\title{
Severe Health Outcomes in Adults with Respiratory Syncytial Virus-associated Hospitalizations
}

\author{
Connor Goldman ${ }^{1}$, William Sieling ${ }^{1}$, Luis Alba ${ }^{1}$, Raul Silverio Francisco ${ }^{1}$, Celibell $\operatorname{Vargas}^{1}$, \\ Angela Barrett ${ }^{1}$, Matthew Phillips ${ }^{2}$, Lyn Finelli ${ }^{2}$, and Lisa Saiman ${ }^{1}$ \\ ${ }^{1}$ Columbia University Irving Medical Center \\ ${ }^{2}$ Merck \& Co Inc
}

September 11, 2020

\begin{abstract}
Background: A systematic assessment of severe clinical outcomes associated with respiratory syncytial virus (RSV) infections in adults is lacking. Methods: Within a prospective surveillance study, we performed a nested retrospective study during two respiratory viral seasons, October 2017-April 2018 and October 2018-April 2019, to determine the proportion of patients with laboratory-confirmed RSV infection who experienced severe outcomes defined as intensive care unit admission, mechanical ventilation, and/or death. We assessed factors associated with these severe outcomes. We explored the impact of RSV-associated hospitalizations on changes in the living situations of surviving patients from admission to discharge. Results: Overall, 403 patients were studied (median age 69 years); $29.5 \%$ were $>80$ years. Common comorbidities included cardiac (47.6\%) and pulmonary $(45.9 \%)$ conditions and diabetes (41.4\%). Severe outcomes occurred in $19.1 \%$ of patients including ICU admissions (16.4\%), mechanical ventilation (12.4\%), and/or death (6.7\%). Patients admitted from residential living facilities had 4.43 times higher likelihood of severe RSV infection compared to those living in the community with or without assistance from family or home health aides. After discharge, 56 (15.1\%) patients required an increased level of support including 36 (9.7\%) with new admissions to residential living facilities. Conclusions: RSV infection was associated with severe illness in adults. Living in a facility was a risk factor for severe outcomes and likely a surrogate for frailty at admission rather than an independent risk factor. The need for an increased level of support after discharge will add RSV-related healthcare costs to those already incurred from hospitalization.
\end{abstract}

\section{INTRODUCTION}

Respiratory syncytial virus (RSV) causes significant morbidity in older adults. It has been estimated that RSV infects $3 \%$ to $10 \%$ of adults each year ${ }^{1}$ and contributes to more than 14,000 adult deaths annually in the United States. ${ }^{2}$ Persons with chronic heart or lung disease and those with immunocompromising conditions appear to be at an increased risk of severe illness from RSV infection. ${ }^{3,4}$ National mortality and viral surveillance data for respiratory and circulatory deaths estimated that $78 \%$ of RSV-associated deaths occurred in persons older than 65 years of age ${ }^{5}$ suggesting age is also a risk factor for mortality. ${ }^{7}$ However, the associations between risk factors such as older age, obesity, and heart, lung, and neurological comorbid conditions and severe outcomes during hospitalization are better understood for influenza than for RSV infection. ${ }^{4}$

As the RSV season typically coincides with seasonal influenza, generally from October to May, ${ }^{6}$ and both viruses can cause lower respiratory tract infections and pneumonia, it can be difficult to ascertain the relative contribution of RSV without comprehensive screening and testing for both viruses. ${ }^{7}$ We performed this nested retrospective study using data derived from a prospective surveillance study for RSV-related hospitalizations in which patients with symptoms of respiratory illness were routinely tested for respiratory viruses. In the 
current study, we identified hospitalized adults with severe outcomes associated with RSV defined as intensive care unit (ICU) admission, mechanical ventilation, and/or in-hospital death. We compared the demographic characteristics, clinical factors, and living situations on admission of patients with and without severe RSV infection. We hypothesized that even among patients with the same comorbidities, baseline living situation could serve as an indicator of a patient's underlying health status. Finally, to assess changes in living situations, we explored the impact of RSV-associated hospitalizations on the type of care and the level of care of surviving patients from hospital admission to discharge.

\section{METHODS}

\section{Study Design, Sites, and Participants}

From October 2017 to April 2018 (Year 1) and from October 2018 to April 2019 (Year 2), we performed prospective surveillance among hospitalized adults 18 years of age and older who had laboratory-confirmed RSV infection. Surveillance occurred at three academically affiliated hospitals from the same multi-campus institution. The hospitals were located in Northern Manhattan and included a 750 -bed tertiary care hospital for adult patients, a 200-bed community hospital for adult patients, and a $\sim 250$-bed children's hospital. The Columbia University Irving Medical Center Institutional Review Board approved this study with a waiver of documentation of consent.

As per the standard of care in these hospitals, patients with acute respiratory infections (ARI) and anticipated hospitalization are tested for viral respiratory pathogens to inform infection prevention and control and influenza treatment. These tests are most often performed in the emergency department (ED). Nasopharyngeal swabs are processed in the clinical microbiology laboratories affiliated with these hospitals using a multiplex reverse transcription polymerase chain reaction (PCR) assay (FilmArray Respiratory Panel, BioFire Diagnostics, Inc., Salt Lake City, UT) which includes RSV on the panel. Potential patients were identified during the prospective surveillance study by monitoring admissions from the ED (Monday through Friday) and by reviewing the Department of Infection Prevention and Control's internal database of epidemiologically significant pathogens in hospitalized patients. Since surveillance did not occur during weekends or holidays, at the end of each surveillance period, the electronic medical records (EMR) of all hospitalized adults were queried to identify any additional patients with positive RSV tests who may have been missed by active surveillance.

Eligible patients for the current study were 18 years and older, had laboratory-confirmed RSV infection, two or more symptoms consistent with ARI (fever, cough, shortness of breath, sore throat, headache, malaise, myalgia, sputum production, wheezing, hoarseness, and/or pleuritic chest pain) documented in the EMR, were hospitalized for at least 24 hours, and lived in New York City as per their residential zip code.

\section{Study Outcomes and Risk Factors Associated with Severe RSV Infection}

The primary outcome was the proportion of patients with severe RSV outcomes, defined as being admitted to an ICU, receiving mechanical ventilation, and/or dying during the RSV-associated hospitalization. The Centers for Disease Control and Prevention National Notifiable Diseases Surveillance System case definition for RSV-related death was used. ${ }^{14}$

Potential factors associated with severe RSV infection were assessed and included demographic characteristics, comorbid conditions, study hospital, study year, and living situation at the time of admission as described in the EMR. The baseline living situations of patients were stratified by implied level of functional independence. Level 1 included patients living without assistance in the community; Level 2 included those living in the community with the assistance of friends, family, and/or a home health aide; and Level 3 included those living in a facility such as assisted living, a skilled nursing or long-term care facility, rehabilitation facility, or hospice. Patients who reported being homeless on admission or were transferred from another acute care facility were excluded from this analysis.

Among the patients who survived and were discharged from the hospital, we explored changes in living situation from admission to discharge that implied the need for a higher level of care at discharge. For 
example, living with assistance on admission (Level 2) and discharge to a skilled nursing home (Level 3) represented the need for a higher level of care. Patients who were discharged to another acute care facility were excluded from this analysis.

\section{Data Collection and Analysis}

Data for outcomes and factors associated with severe RSV infection were extracted from the EMR and entered into standardized electronic case report forms maintained on a secure server.

Chi-squared test of proportions was utilized to determine differences in the distribution of severe illness by month and by surveillance year. Odds ratios (OR) and $95 \%$ confidence intervals (CI) were used to estimate the odds of severe RSV outcomes by demographic characteristics, comorbid conditions, and living situation. We built a multivariate logistic regression model using bidirectional elimination to evaluate characteristics associated with severe RSV outcomes. Variables included in the model were those hypothesized to be associated with severe illness, i.e., older age, obesity, and heart, lung, and neurologic comorbidities and any factors significantly associated with severe RSV infection in the bivariate analysis. The final model was selected by minimizing the Akaike information criterion (AIC) and maximizing the coefficient of determination $\left(\mathrm{r}^{2}\right)$.

We explored whether severe RSV outcomes impacted the discharge level of care needed, using living situation as a surrogate for the level of care. To do so, we compared the pre-admission and discharge living situation for each surviving patient. Changes in the patient's living situation were categorized as increased (e.g., living independently before hospitalization and discharged to a nursing home), decreased (e.g., living in the community with family before hospitalization and discharged to living independently), or unchanged level of care. Odds ratios and $95 \%$ confidence intervals were used to estimate the odds of change in living situation among those with and without severe RSV outcomes.

Fisher's Exact test was utilized, when appropriate. A p-value of $<0.05$ was considered statistically significant. R-Studio (https://rstudio.com, Version 1.2.5001) packages and procedures were used for data analysis.

\section{RESULTS}

\section{Study Population}

During the two-year study period, 403 patients fulfilled the eligibility criteria for RSV-associated hospitalization. The median age was 69 years (Interquartile range [IQR] 57.2-82.1 years), 29.5\% were 80 years of age and older, and $58.3 \%$ were female (Table 1 ). The most common comorbid conditions were cardiac conditions $(47.6 \%)$, respiratory conditions $(45.9 \%)$, and diabetes (41.4\%). Only $4.7 \%$ of patients had no comorbid conditions. Fewer than half (41.9\%) were living independently at admission.

\section{Patients with Severe RSV Outcomes}

Overall, 77 (19.1\%) of the 403 patients experienced severe illness including 66 (16.4\%) who were admitted to an ICU, 50 (12.4\%) who received mechanical ventilation, and/or 27 (6.7\%) who died during hospitalization. The median length of ICU stay was seven days (IQR 3.5 to 12 days) and the median duration of mechanical ventilation was three days (IQR 2 to 9.5 days).

The number of patients hospitalized with laboratory-confirmed RSV infection ranged from 4-92 each month and the number of patients with severe illness ranged from 0-21 each month (Figure 1). Both surveillance years, the number of patients hospitalized with RSV infection was highest in December and January. The proportion of patients with severe RSV outcomes each year and each month were similar.

\section{Factors Associated with Severe RSV Outcomes}

Factors associated with severe RSV outcomes were assessed with bivariate analysis (Table 1). Neither age nor any of the comorbid conditions assessed were associated with increased odds of experiencing severe RSV infection. Patients who resided in assisted living, skilled nursing, long-term care, rehabilitation, or hospice facilities at admission (Level 3) had a 4.43 times higher likelihood of severe RSV outcomes compared with other living situations. 
The results from the logistic regression analysis are shown in Table 2. Multiple models were tested, including age stratified models assessing multiple different comorbidities as well as clinically and statistically significant variables from the bivariate analysis. In the final model, those with a living situation of Level 3 had 6.64 times higher odds of severe RSV infection compared to those admitted from other living situations, while controlling for age, sex, and comorbidities.

\section{Changes in Living Situation from Admission to Discharge}

The changes in living situation for the 372 surviving patients who were discharged are shown in Table 3 . Patients who returned to living independently in the community (Level $1, \mathrm{n}=127$ ) or returned to living in the community with assistance (Level $2, \mathrm{n}=156)$ were less likely to have had severe RSV outcomes $(\mathrm{OR}=0.48$ and 0.33 respectively). Patients who lived in a facility and returned to a facility (Level $3, \mathrm{n}=30$ ) were 4.92 times more likely to have had severe RSV outcomes. Severe RSV outcomes were significantly more likely to have occurred among patients who required an increased level of care at discharge including those whose specific level of care increased from Level 1 to Level $3(\mathrm{OR}=5.41)$ or from Level 2 to Level $3(\mathrm{OR}=3.17)$.

\section{CONCLUSIONS}

We found that $19 \%$ of patients with RSV-associated hospitalization experienced severe outcomes, including $6.7 \%$ who died during hospitalization. The proportion of deaths associated with RSV infection is consistent with previous studies of similar populations $\left(8 \%^{1}, 5.6 \%^{4}\right)$. ICU admission and/or mechanical ventilation have been shown to be associated with increased morbidity and mortality, longer lengths of hospital stay as well as hospital-associated infections. ${ }^{9}$ Furthermore, ICU admissions were found to be three to four times more costly than admissions to non-ICU units. ${ }^{10}$

Previous studies have compared morbidity and mortality in adult patients with RSV-related and influenzarelated hospitalizations. ${ }^{11,12}$ Hospitalized adults in Hong Kong with RSV infection had comparable 30- and 60-day mortality compared to those with influenza, but significantly higher rates of mechanical ventilation ( $11.1 \%$ vs. $6.2 \%, \mathrm{p}=0.003)$, and extended care in subacute hospitals following discharge $(25.2 \%$ vs. $19.7 \%$, $\mathrm{p}=0.027) .{ }^{11}$ Analysis of Medicaid data in Tennessee found that RSV infection and influenza accounted for a similar estimated number of hospitalizations. ${ }^{12}$

We found that nearly half of the patients admitted from assisted living, a skilled nursing or long-term care facility, rehabilitation facility, or hospice had severe RSV outcomes. While age and specific comorbidities were not associated with severe disease, those living in a facility prior to admission had 4.43 times the odds of severe disease compared to those living independently or with assistance in the community. Similarly, when controlling for age and comorbidities, those who lived in a facility had 6.64 times the odds of severe RSV infection compared to those living independently or with assistance. These findings suggest that living situation may be a proxy measure of frailty and, in turn, a risk for severe disease. ${ }^{13}$ Chronic disease, multiple comorbidities, and functional disability are associated with frailty, and frailty has been shown to contribute to older adults' vulnerability to adverse outcomes from influenza. ${ }^{14,15}$ Other studies have shown that those in assisted living have higher rates of frailty, ${ }^{16}$ although it should be noted that there are inconsistent measures of frailty. Future interventions for RSV such as vaccines or antiviral therapy should target this vulnerable high-risk population.

Furthermore, we found that $15.1 \%$ of adults who survived hospitalization required an increased level of care as reflected in their living situation at discharge when compared with that on admission. Overall, 36 patients $(9.7 \%)$ went from living in the community to living in a facility. Other studies have shown that hospitalization is an independent risk factor for declines in functional status among older adults which can be associated with a need for increased level of care. ${ }^{17}$ The yearly median cost of assisted living was estimated to be $\$ 48,612$ in $2019 .{ }^{18}$ Thus, studies of healthcare costs associated with RSV hospitalizations should include those incurred after discharge as the need for an increased level of support after discharge will add RSV-related healthcare costs to those already incurred from hospitalization.

This study had limitations. This study was performed in an urban, academically affiliated medical center 
and the findings may not be generalizable. Although patients had laboratory-confirmed RSV infection and at least two symptoms of ARI, their outcomes, including severe outcomes, may have been secondary to exacerbations of their comorbid conditions. Assessment of comorbidities were collected as reported in the admission notes, but were not verified by treatment, did not account for disease severity, or management during hospitalization. Living situation was self-reported or reported by a family member and not otherwise verified. Living situation might not be due to health-related issues, e.g., patients could be living with a family member due to financial constraints or to assist with childcare.

There are currently no specific therapies or approved vaccines for prevention of RSV infection in adults. These data further highlight the need for prevention strategies for RSV infection in older populations, especially those living in residential living facilities. While frail, older adults may have a diminished capacity to respond to vaccines, prevention of RSV transmission in congregate settings is highly desirable and feasible. Infection prevention strategies combined with vaccination could reduce morbidity and mortality as well as healthcare costs. Future studies should further assess the association of living situation and adverse outcomes as well as the association of RSV-related hospitalizations and longer-term deterioration in functional status.

\section{References}

1. Falsey AR, Hennessey PA, Formica MA, Cox C, Walsh EE. Respiratory syncytial virus infection in elderly and high-risk adults. N Engl J Med. 2005;352(17):1749-1759. doi:10.1056/NEJMoa043951

2. NNDSS. Respiratory Syncytial Virus-Associated Mortality (RSV-Associated Mortality). $\quad$ https://wwwn.cdc.gov/nndss/conditions/respiratory-syncytial-virus-associated-mortality/casedefinition/2019/ Accessed April 26, 2020

3. CDC. RSV in Older Adults and Adults with Chronic Medical Conditions. https://www.cdc.gov/rsv/highrisk/older-adults.html Accessed April 26, 2020

4. Ackerson B, Tseng HF, Sy LS, et al. Severe morbidity and mortality associated with respiratory syncytial virus versus influenza infection in hospitalized older adults. Clin Infect Dis. 2019;69(2):197-203. doi:10.1093/cid/ciy991

5. Thompson WW. Mortality associated with influenza and respiratory syncytial virus in the United States. JAMA. 2003;289(2):179. doi:10.1001/jama.289.2.179

6. Rose EB, Wheatley A, Langley G, Gerber S, Haynes A. Respiratory syncytial virus seasonality — United States, 2014-2017. MMWR Morb Mortal Wkly Rep. 2018;67(2):71-76. doi:10.15585/mmwr.mm6702a4

7. Piedimonte G, Perez MK. Respiratory syncytial virus infection and bronchiolitis. Pediatr Rev. 2014;35(12):519-530. doi:10.1542/pir.35-12-519

8. CDC. Respiratory Syncytial Virus-Associated Mortality (RSV-Associated Mortality) 2019 Case Definition. https://wwwn.cdc.gov/nndss/conditions/respiratory-syncytial-virus-associated-mortality/casedefinition/2019/ Accessed April 27, 2020

9. Hazra A, Dasgupta S, Das S, Chawan NS. Nosocomial infections in the intensive care unit: Incidence, risk factors, outcome and associated pathogens in a public tertiary teaching hospital of Eastern India. Indian J Crit Care Med. 2015;19(1):14-20. doi:10.4103/0972-5229.148633

10. Gyldmark M. A review of cost studies of intensive care units Problems with the cost concept. Crit Care Med. 1995;23(5):964-972.

11. Lee N, Lui GCY, Wong KT, et al. High morbidity and mortality in adults hospitalized for respiratory syncytial virus infections. Clin Infect Dis. 2013;57(8):1069-1077. doi:10.1093/cid/cit471

12. Griffin MR, Coffey CS, Neuzil KM, Mitchel EF, Wright PF, Edwards KM. Winter viruses: influenza- and respiratory syncytial virus-related morbidity in chronic lung disease. Arch Intern Med. 2002;162(11):1229. doi:10.1001/archinte.162.11.1229 
13. Leng S, Chen X, Mao G. Frailty syndrome: an overview. Clin Interv Aging. Published online March 2014:433. doi:10.2147/CIA.S45300

14. McElhaney JE, Zhou X, Talbot HK, et al. The unmet need in the elderly: How immunosenescence, CMV infection, co-morbidities and frailty are a challenge for the development of more effective influenza vaccines. Vaccine. 2012;30(12):2060-2067. doi:10.1016/j.vaccine.2012.01.015

15. Lees C, Godin J, McElhaney JE, et al. Frailty hinders recovery from influenza and acute respiratory illness in older adults. J Infect Dis. Published online March 9, 2020:jiaa092. doi:10.1093/infdis/jiaa092

16. Lutomski JE, Baars MAE, Boter H, et al. Frailty, disability and multi-morbidity: the relationship with quality of life and healthcare costs in elderly people. Ned Tijdschr Geneeskd. 2014;158:A7297.

17. Boyd CM, Landefeld CS, Counsell SR, et al. Recovery of activities of daily living in older adults after hospitalization for acute medical illness: FUNCTIONAL RECOVERY AFTER HOSPITALIZATION. J Am Geriatr Soc. 2008;56(12):2171-2179. doi:10.1111/j.1532-5415.2008.02023.x

18. Genworth. Cost of Care Survey. https://www.genworth.com/aging-and-you/finances/cost-of-care.html Accessed June 25, 2020

Table 1: Comparison of Demographic and Clinical Factors in Hospitalized Adult Subjects with and without Severe RSV Outcomes

\begin{tabular}{llll}
\hline Factors & All & Without Severe RSV Outcomes & With Severe RSV Outcom \\
\hline & $\mathrm{N}=403$ & $\mathrm{n}=326$ & $\mathrm{n}=77$ \\
Median age (IQR) years & $69.0(57.2-82.1)$ & $69.3(56.4-81.9)$ & $68.4(57.9-82.3)$ \\
Age strata & & & \\
18-49 years & $62(15.4 \%)$ & $47(14.4 \%)$ & $15(19.5 \%)$ \\
$50-64$ years & $110(27.3 \%)$ & $96(29.4 \%)$ & $14(18.2 \%)$ \\
65-79 years & $112(27.8 \%)$ & $88(27 \%)$ & $24(31.2 \%)$ \\
>80 years & $119(29.5 \%)$ & $95(29.1 \%)$ & $24(31.2 \%)$ \\
Sex & & & $44(57.1 \%)$ \\
Female & $235(58.3 \%)$ & $201(61.7 \%)$ & $17(22.1 \%)$ \\
Race & & & $19(24.7 \%)$ \\
White & $106(26.3 \%)$ & $89(27.3 \%)$ & $0(0 \%)$ \\
Black or African American & $80(19.9 \%)$ & $61(18.7 \%)$ & $0(0 \%)$ \\
Asian & $5(1.2 \%)$ & $5(1.5 \%)$ & $41(53.2 \%)$ \\
Other & $8(2.0 \%)$ & $8(2.5 \%)$ & $19(24.7 \%)$ \\
Unknown & $204(50.6 \%)$ & $163(50 \%)$ & $16(20.8 \%)$ \\
Ethnicity & & & $42(54.5 \%)$ \\
Hispanic & $129(32.0 \%)$ & $110(33.7 \%)$ & \\
Non-Hispanic & $73(18.1 \%)$ & $57(17.5 \%)$ & $33(42.9 \%)$ \\
Unknown & $201(49.9 \%)$ & $159(48.8 \%)$ & $23(30.0 \%)$ \\
Comorbid Conditions & & & $38(49.4 \%)$ \\
Any Cardiac & $192(47.6 \%)$ & $159(48.8 \%)$ & $24(31.2 \%)$ \\
CHF & $111(27.5 \%)$ & $88(27.0 \%)$ & $21(27.3 \%)$ \\
Any Respiratory & $185(45.9 \%)$ & $147(45.1 \%)$ & $34(44.2 \%)$ \\
COPD & $91(22.6 \%)$ & $67(20.6 \%)$ & $20(26.0 \%)$ \\
Asthma & $103(25.6 \%)$ & $82(25.2 \%)$ & $16(20.8 \%)$ \\
Diabetes & $167(41.4 \%)$ & $133(40.8 \%)$ & \\
Any Neurologic & $113(28.0 \%)$ & $93(28.5 \%)$ & $91(27.9 \%)$ \\
Obesity & $107(26.6 \%)$ & $83(25.5 \%)$ & \\
Any Immunosuppressive & $101(25.1 \%)$ & & \\
& & &
\end{tabular}




\begin{tabular}{llll}
\hline Factors & All & Without Severe RSV Outcomes & With Severe RSV Outcom \\
\hline None & $19(4.7 \%)$ & $14(4.3 \%)$ & $5(6.5 \%)$ \\
Hospital & & & \\
A & $289(71.7 \%)$ & $235(72.1 \%)$ & $54(70.1 \%)$ \\
B & $107(26.6 \%)$ & $85(26.1 \%)$ & $22(28.6 \%)$ \\
C Study Year & $7(1.7 \%)$ & $6(1.8 \%)$ & $1(1.3 \%)$ \\
1 & & & \\
2 & $184(45.7 \%)$ & $151(46.3 \%)$ & $33(42.9 \%)$ \\
Admission living situation ++ & $219(54.3 \%)$ & $175(53.7 \%)$ & \\
Level 1 & & & $28(37.1 \%)$ \\
Level 2 & $169(41.9 \%)$ & $141(43.3 \%)$ & $29(37.7 \%)$ \\
Level 3 & $193(47.9 \%)$ & $164(50.3 \%)$ & $18(23.4 \%)$ \\
\hline
\end{tabular}

${ }^{+}$Fischer's Exact Test

${ }^{++}$Two patients were excluded from analysis of living situation; one was homeless at admission and one was transferred from another hospital

Table 2: Regression Analysis for Factors Associated with Severe RSV Outcomes in Hospitalized Adults

\begin{tabular}{llll}
\hline Variable $^{+}$ & Estimated OR $[\mathbf{C I}]$ & $\beta$ & $\mathbf{P r}(>|\mathbf{z}|)$ \\
\hline Intercept & $0.15[0.07,0.31]$ & -1.92 & $<\mathbf{0 . 0 0 1}$ \\
Age 18-49 & $1.73[0.75,4.00]$ & 0.55 & 0.19602 \\
Age 50-64 & Reference Group & Reference Group & Reference Group \\
Age 65-79 & $1.76[0.86,3.61]$ & 0.57 & 0.121306 \\
Age 80+ & $1.25[0.58,2.70]$ & 0.23 & 0.559866 \\
Sex (Male) & $1.20[0.70,2.07]$ & 0.18 & 0.510641 \\
Respiratory Condition & $1.36[0.80,2.33]$ & 0.31 & 0.261441 \\
Cardiac Condition & $0.73[0.42,1.27]$ & -0.31 & 0.266339 \\
Immunosuppressive Condition & $0.89[0.48,1.68]$ & -0.11 & 0.736981 \\
Neurologic Condition & $0.60[0.30,1.18]$ & -0.51 & 0.135876 \\
Living in a facility at admission (Level 3) & $6.64[2.92,15.08]$ & 1.89 & $<\mathbf{0 . 0 0 1}$ \\
\hline
\end{tabular}

+ The final model is as shown: Logit(sRSV) $=$ intercept $+\beta_{1}$ (Age 18-49) $+\beta_{2}$ (Age 65-79) $+\beta_{3}$ (Age $80+)+\beta_{4}($ male $)+\beta_{5}($ Lung Condition $)+\beta_{6}$ (Cardio Condition $)+\beta_{7}($ Immunosuppressive Condition $)+$ $\beta_{8}$ (Neurological Condition) $+\beta_{9}$ (living in a facility at admission). Final $\mathrm{R}^{2}=0.098 ; \mathrm{AIC}=384.47$

Table 3: Changes in Living Situation from Admission to Discharge among Hospitalized Adults with RSV

\begin{tabular}{llllll}
\hline $\begin{array}{l}\text { Change from } \\
\text { Admission to }\end{array}$ & $\begin{array}{l}\text { Total } \\
(\mathbf{n = 3 7 2})^{++}\end{array}$ & $\begin{array}{l}\text { Without } \\
\text { Severe RSV } \\
\text { Outcomes } \\
(\mathbf{n = 3 2 5})\end{array}$ & $\begin{array}{l}\text { With Severe } \\
\text { RSV } \\
\text { Outcomes } \\
(\mathbf{n}=\mathbf{4 7})\end{array}$ & OR CI $\mathbf{9 5}$ & P-value \\
\hline $\begin{array}{l}\text { Discharge } \\
\text { Unchanged: }\end{array}$ & $127(34.1 \%)$ & $117(36.0 \%)$ & $10(21.3 \%)$ & $0.48(0.23$, & $\mathbf{0 . 0 5 0}$ \\
Level 1 -> & & & & $1.00)$ & \\
Level 1 & & & &
\end{tabular}




\begin{tabular}{|c|c|c|c|c|c|}
\hline $\begin{array}{l}\text { Change from } \\
\text { Admission to } \\
\text { Discharge }^{+}\end{array}$ & $\begin{array}{l}\text { Total } \\
(\mathrm{n}=372)^{++}\end{array}$ & $\begin{array}{l}\text { Without } \\
\text { Severe RSV } \\
\text { Outcomes } \\
(n=325)\end{array}$ & $\begin{array}{l}\text { With Severe } \\
\text { RSV } \\
\text { Outcomes } \\
(n=47)\end{array}$ & OR CI 95 & $P$ - value \\
\hline $\begin{array}{l}\text { Unchanged: } \\
\text { Level } 2 \text {-> } \\
\text { Level } 2\end{array}$ & $156(41.9 \%)$ & $146(44.9 \%)$ & $10(21.3 \%)$ & $\begin{array}{l}0.33(0.16, \\
0.69)\end{array}$ & 0.003 \\
\hline $\begin{array}{l}\text { Unchanged: } \\
\text { Level } 3 \text {-> } \\
\text { Level } 3\end{array}$ & $30(8.1 \%)$ & $19(5.8 \%)$ & $11(23.4 \%)$ & $\begin{array}{l}4.92(2.17 \\
11.16)\end{array}$ & $<0.001$ \\
\hline $\begin{array}{l}\text { Increased: } \\
\text { Level } 1 \text {-> } \\
\text { Level } 2\end{array}$ & $20(5.4 \%)$ & $17(5.2 \%)$ & $3(6.4 \%)$ & $\begin{array}{l}1.24(0.35 \\
4.39)\end{array}$ & 0.731 \\
\hline $\begin{array}{l}\text { Increased: } \\
\text { Level } 1 \text {-> } \\
\text { Level } 3\end{array}$ & $12(3.2 \%)$ & $7(2.2 \%)$ & $5(10.6 \%)$ & $\begin{array}{l}5.41(1.64, \\
17.81)\end{array}$ & 0.005 \\
\hline $\begin{array}{l}\text { Increased: } \\
\text { Level } 2 \text {-> } \\
\text { Level } 3\end{array}$ & $24(6.5 \%)$ & $17(5.2 \%)$ & $7(14.9 \%)$ & $\begin{array}{l}3.17(1.24, \\
8.12)\end{array}$ & 0.016 \\
\hline $\begin{array}{l}\text { Any increased } \\
\text { level of care }\end{array}$ & $56(15.1 \%)$ & $41(12.6 \%)$ & $15(31.9 \%)$ & $\begin{array}{l}3.25(1.62, \\
6.51)\end{array}$ & 0.001 \\
\hline $\begin{array}{l}\text { Decreased } \\
\text { level of care }\end{array}$ & $3(0.8 \%)$ & $2(0.6 \%)$ & $1(2.1 \%)$ & $\begin{array}{l}3.51(0.31 \\
39.49)\end{array}$ & 0.334 \\
\hline
\end{tabular}

+Level 1: independent; Level 2: assistance of family, friends, or home health-aide; Level 3: assisted living, skilled nursing or long-term facility, rehabilitation facility, or hospice.

${ }^{++}$Analysis included 372 surviving patients and not discharged to another acute care hospital. Excluded patients: one patient who was homeless at admission, three patients discharged to another acute care facility, and 27 patients who died.

$\S$ Patient required lower level of care at discharge compared to at hospitalization

Figure Legend:

Figure 1: Number of patients admitted with and without severe RSV illness by month and surveillance year. Surveillance was conducted from October through April each year. The percent of patient with severe illness each month is shown ( $\mathrm{Y}$ axis on left). The number of patients with and without severe diseases is shown ( $\mathrm{Y}$ axis on right). The numbers over the histogram bars are the number of patients with and without severe disease each month. nsRSV = not severe RSV; sRSV = severe RSV. 


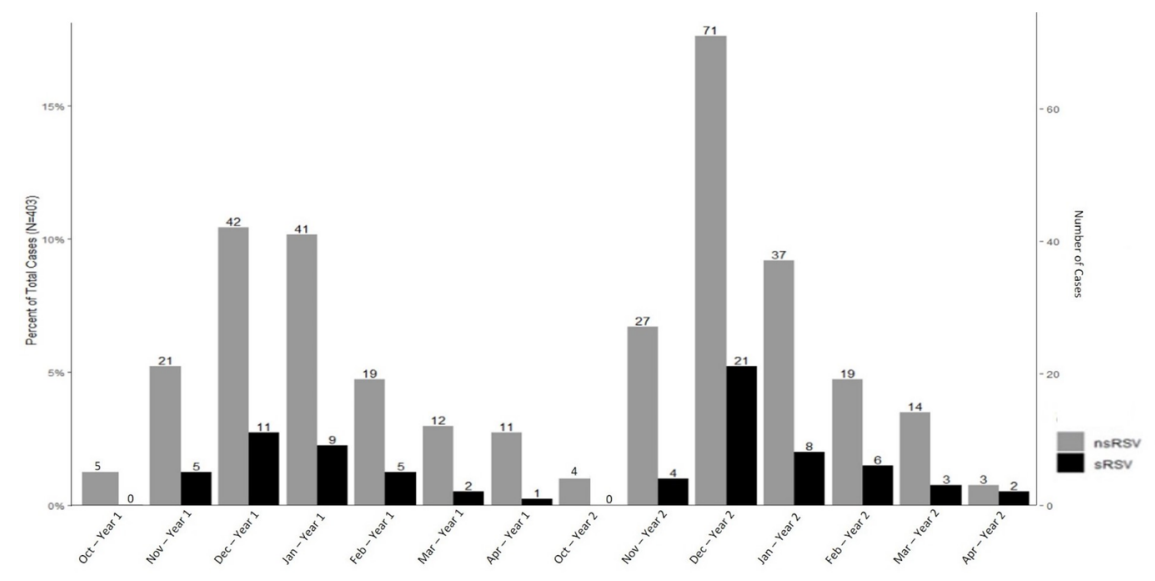

\section{Hosted file}

Goldman et al. Table 1.docx available at https://authorea.com/users/357533/articles/ 480039-severe-health-outcomes-in-adults-with-respiratory-syncytial-virus-associatedhospitalizations

\section{Hosted file}

Goldman et al. Table 2.docx available at https://authorea.com/users/357533/articles/ 480039-severe-health-outcomes-in-adults-with-respiratory-syncytial-virus-associatedhospitalizations

\section{Hosted file}

Goldman et al. Table 3.docx available at https://authorea.com/users/357533/articles/ 480039-severe-health-outcomes-in-adults-with-respiratory-syncytial-virus-associatedhospitalizations 